\title{
Textbooks in Browsers
}

bit.ly / fletcher-bib13

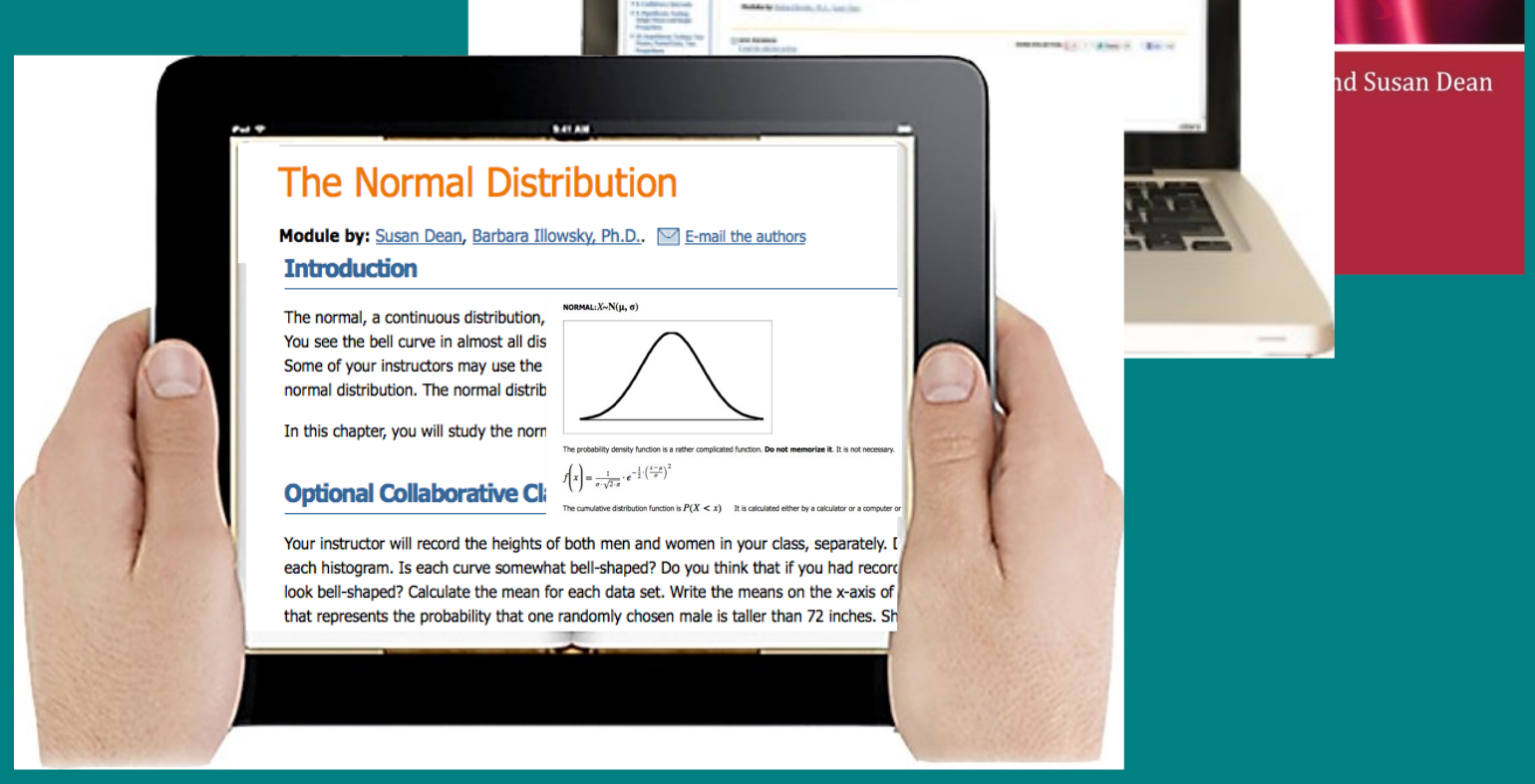




\section{My Background}

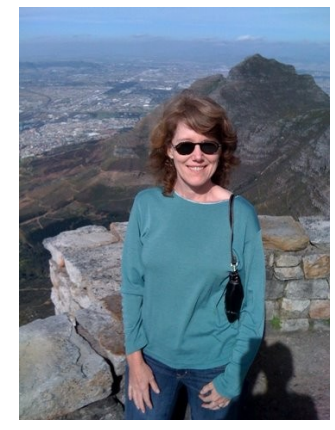

Kathi Fletcher

Connexions PM and Technical Director 4 Yrs Shuttleworth Foundation Fellow 3 Yrs

\section{SHUTTLEWORTH}

FELLOW

\begin{tabular}{|l|l|l|}
\hline O & E & R \\
\hline $\mathbf{P}$ & $\mathbf{U}$ & $\mathbf{B}$ \\
\hline
\end{tabular}

Tools for Remixing Open Education Resources 


\section{Vision for learning}
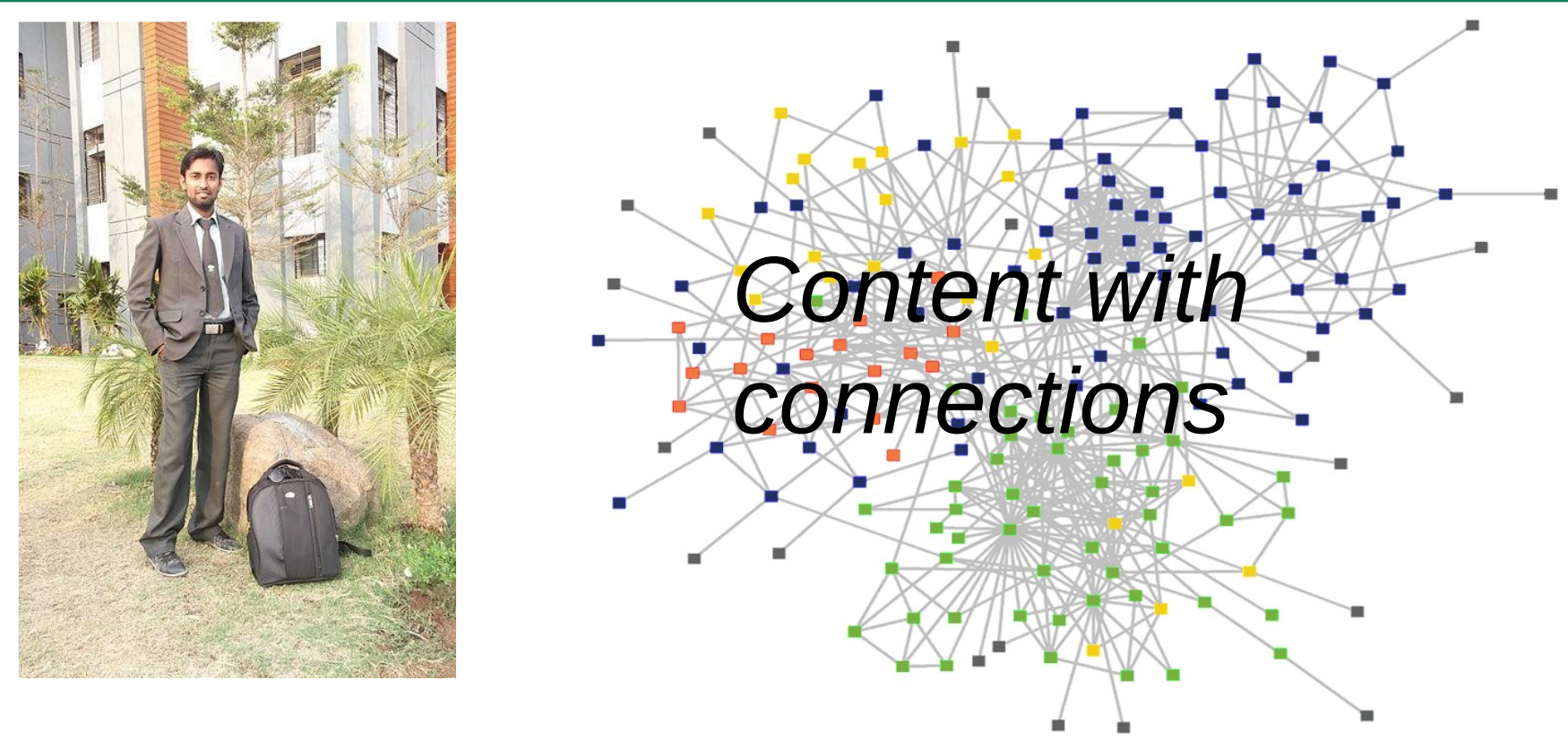

Everywhere I am, on all my devices

My classes and

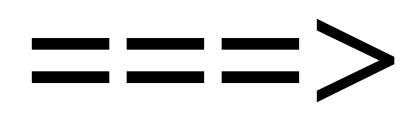
my past knowledge

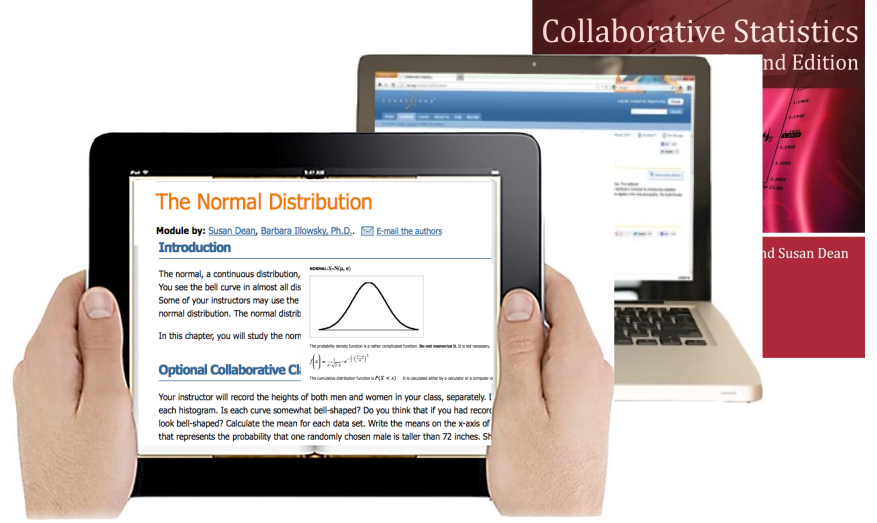




\section{Learning content}

classes, textbooks, articles
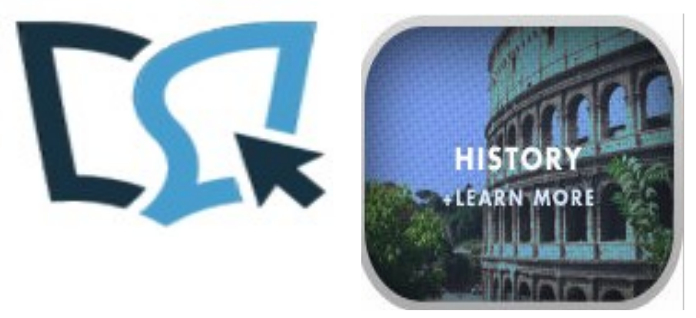

Collabo rative Stats
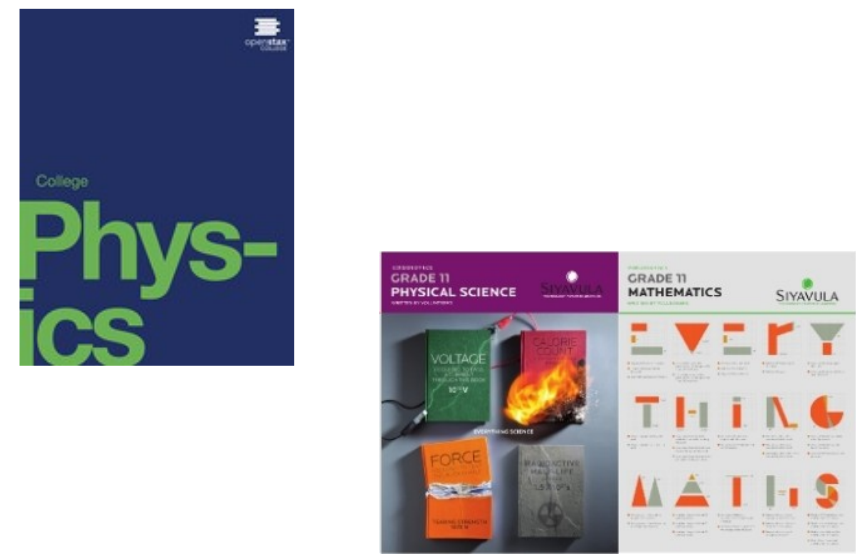

@oerpub @kefletcher 


\section{Transmogrified}

classes, textbooks, articles
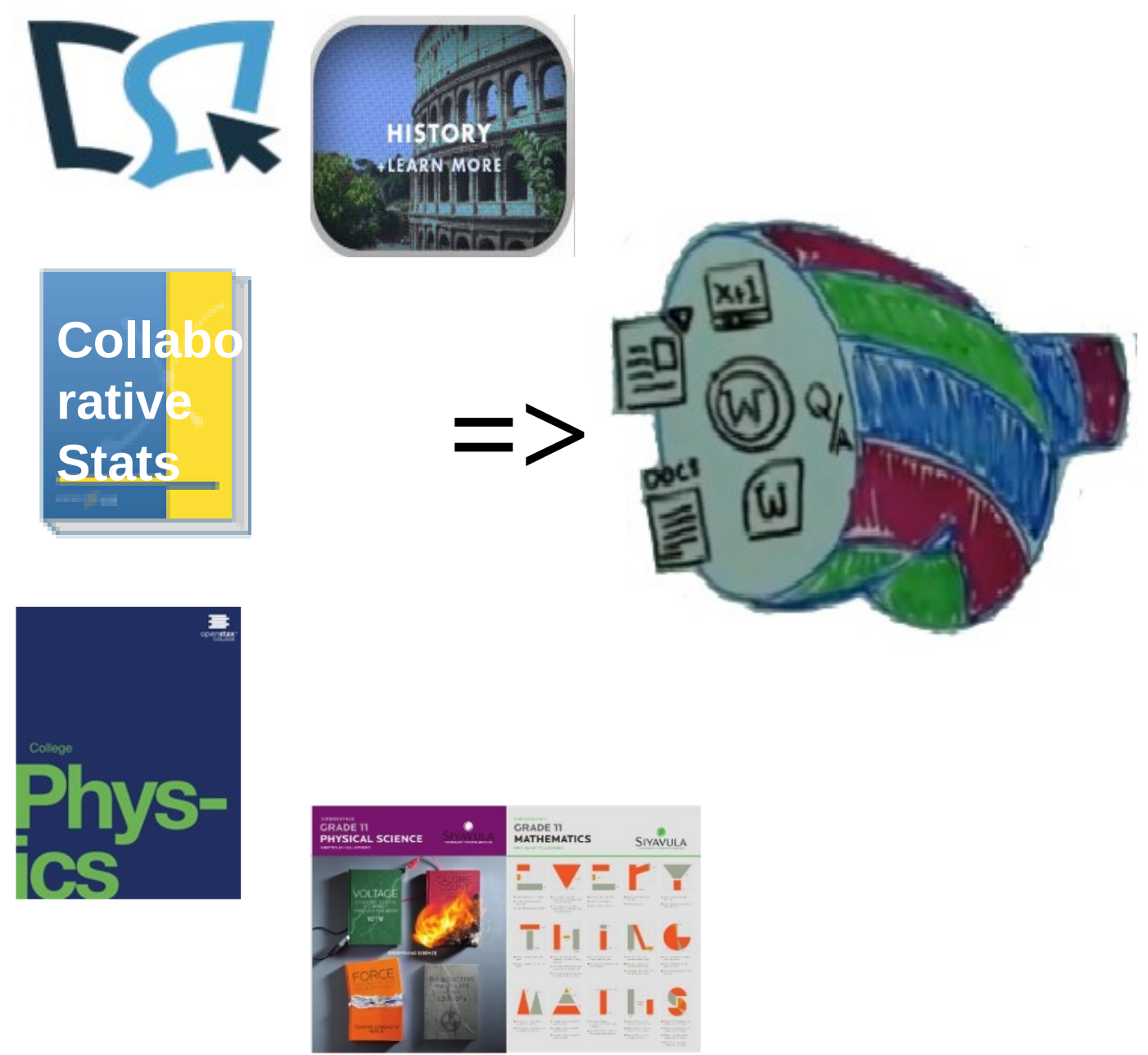

@oerpub @kefletcher 


\section{Everywhere}

classes, textbooks, articles

\section{online, mobile, print}
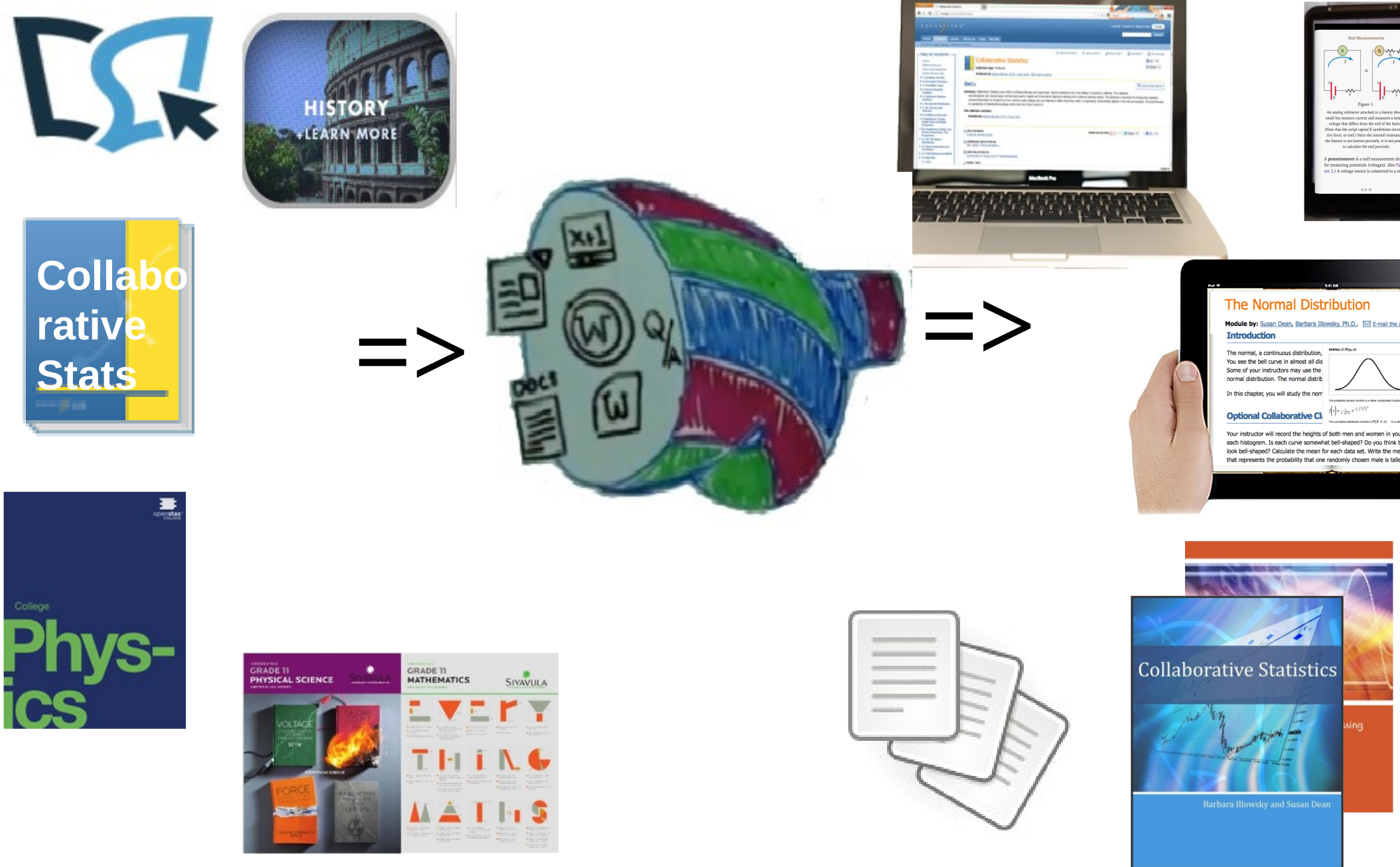

@oerpub @kefletcher http:I/bit.ly/fletcher-bib13 \#bib13

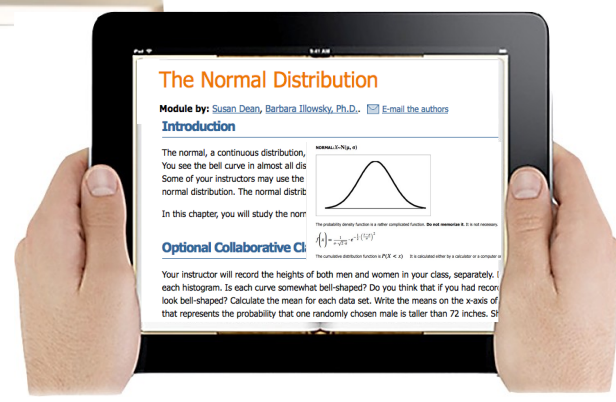




\section{Books \& courses in - flash cards out}
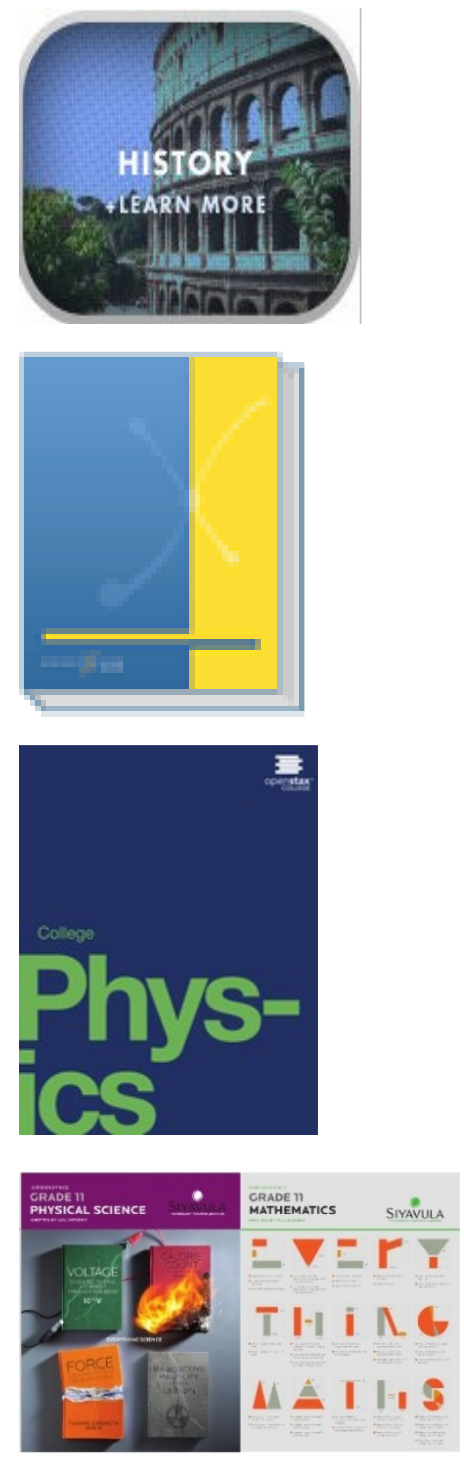
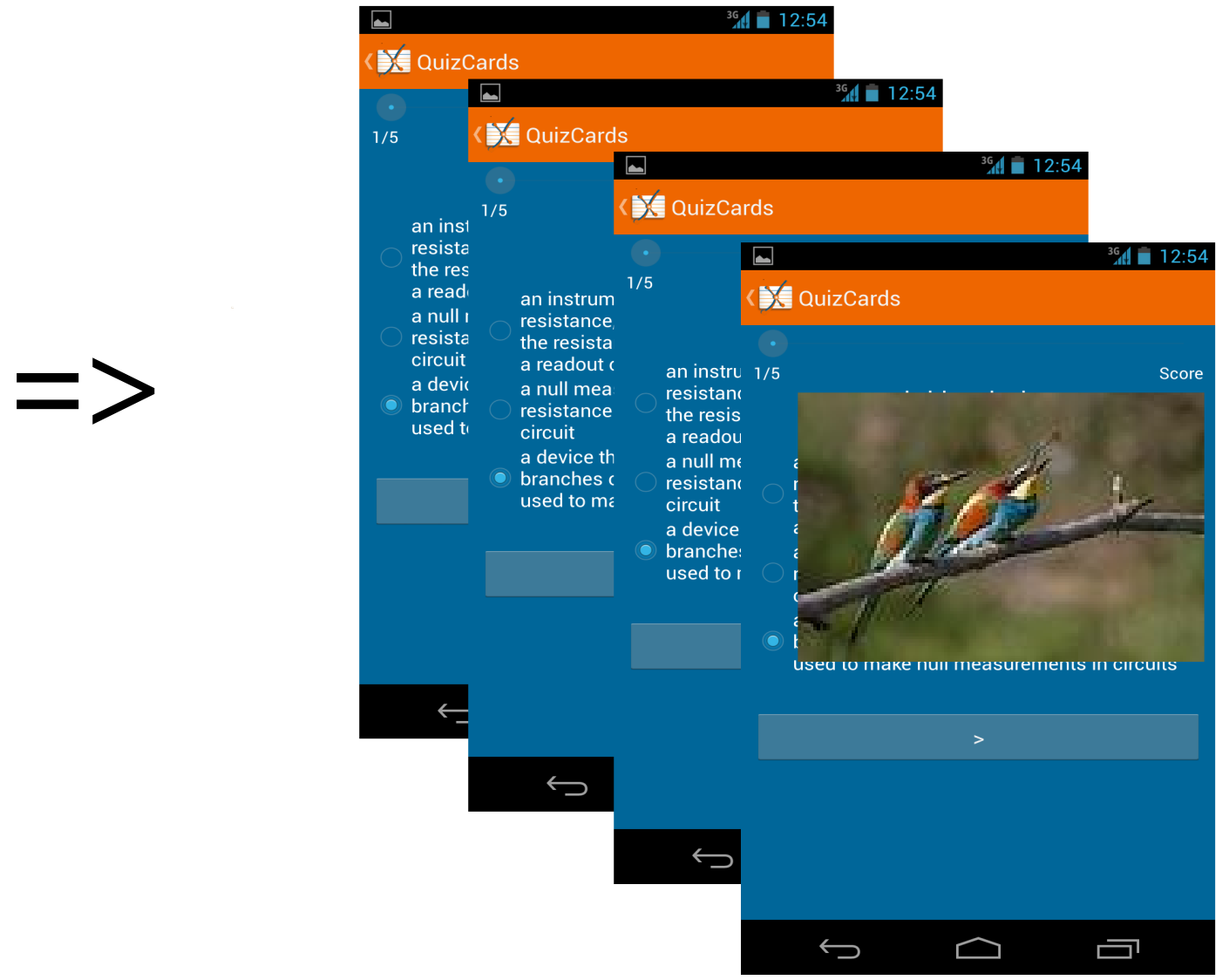

Birds By Pierre Dalous (Own work) [CC-BY-SA-3.0 (http://creativecommons.org/licenses/by-sa/3.0)], via Wikimedia Commons 


\section{Learning content in - practice probs out}
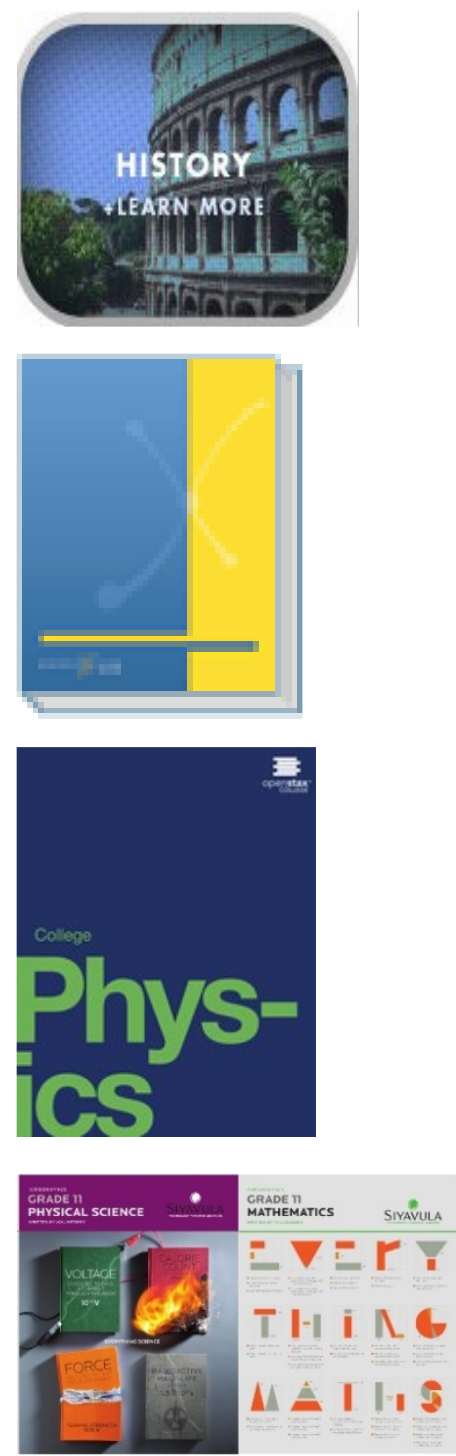
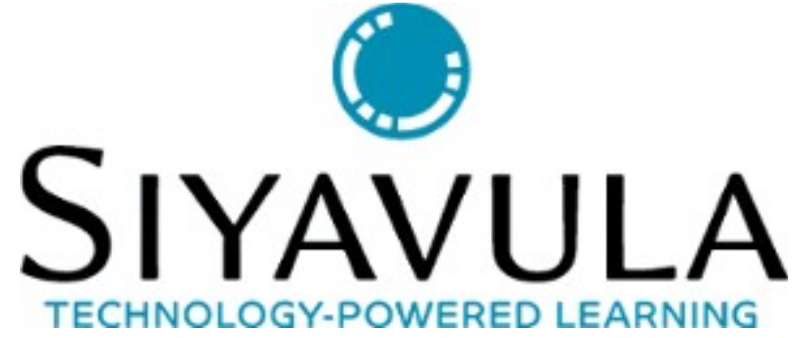

\section{Understanding Ohm's Law}

Consider the circuit which contains one resistor below.
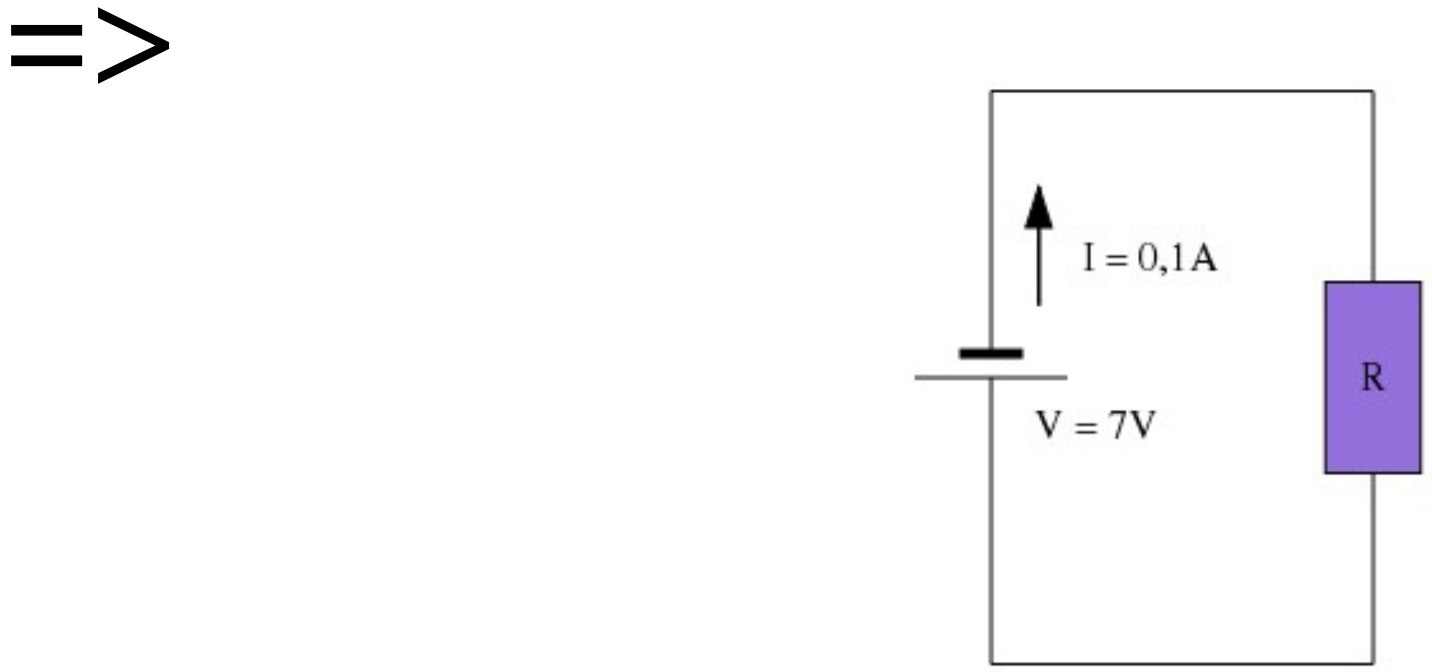

Calculate the resistance $R$ of the resistor. Round your answer to 2 Answer: resistance $=70$ $\Omega \quad[2 / 2$ marks $]$ 


\section{Velocity}

Your notion of velocity is probably the same as its scientific definition. You know that if you have a large displacement in a small amount of time you have a large velocity, and that velocity has units of distance divided by time, such as miles per hour or kilometers per hour.

\section{AVERAGE VELOCITY:}

Average velocity is displacement (change in position) divided by the time of travel,

$$
\bar{v}=\frac{\Delta x}{\Delta t}=\frac{x_{\mathrm{f}}-x_{0}}{t_{\mathrm{f}}-t_{0}},
$$

where $\bar{v}$ is the average (indicated by the bar over the $v$ ) velocity, $\Delta x$ is the change in position (or displacement), and $x_{\mathrm{f}}$ and $x_{0}$ are the final and beginning positions at times $t_{\mathrm{f}}$ and $t_{0}$, respectively. If the starting time $t_{0}$ is taken to be zero, then the average velocity is simply

\section{@oerpub




\section{How? Find learner's notes}

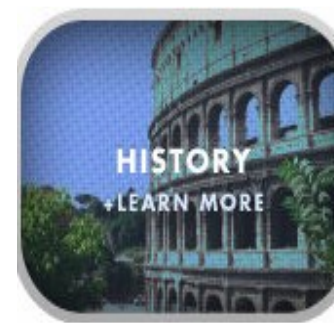

\section{Kathi Fletcher}

11:39 PM Today

Resolve

Carpetbaggers: Northerners who came

to the South after the civil war

Edit Delete

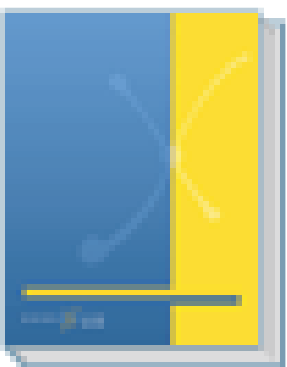

Reply to this comment..

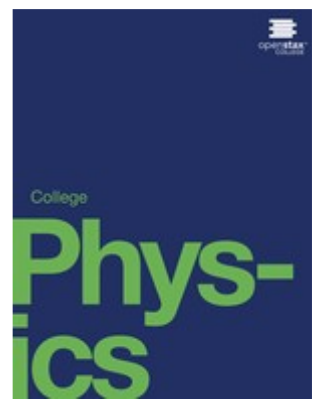

\section{S Kathi Fletcher}

Resolve

$\mathrm{F}=\mathrm{MA}$ Force equals mass times

acceleration

Edit Delete

Reply to this comment..

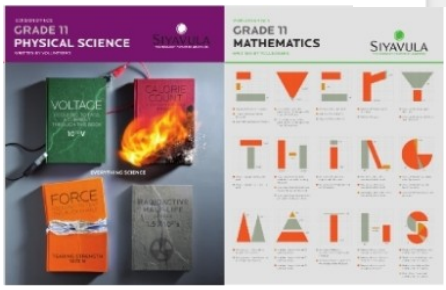




\section{How? Find exercises and problems}

\section{Check Your Understanding}

A commuter train travels from Baltimore to Washington, DC, and back in 1 hour and 45 minutes. The distance between the two stations is approximately 40 miles. What is (a) the average velocity of the train, and (b) the average speed of the train in $\mathrm{m} / \mathrm{s}$ ?

Solution

(a) The average velocity of the train is zero because $x_{\mathrm{f}}=x_{0}$; the train ends up at the same place it starts.

(b) The average speed of the train is calculated below. Note that the train travels 40 miles one way and 40 miles back, for a total distance of 80 miles.

$$
\frac{\text { distance }}{\text { time }}=\frac{80 \text { miles }}{105 \text { minutes }}
$$

$$
\frac{80 \text { miles }}{105 \text { minutes }} \times \frac{5280 \text { feet }}{1 \text { mile }} \times \frac{1 \text { meter }}{3.28 \text { feet }} \times \frac{1 \text { minute }}{60 \text { seconds }}=20 \mathrm{~m} / \mathrm{s}
$$

\section{@oerpub




\section{What is needed to achieve the vision?}

Common Format

- Recognizable structure (definitions, exercises, etc.)

Easy to use editor for authors

Easy to extend

Publicly available books 


\section{All possible now}

- HTML5 (language of the web)

- Separate structure and style

- HTMLBook, TextbookHTML

- EPUB3 (language of mobile web)

- Browser-based technology

- Create, annotate, view on the web

- Content hosts

- Github, Connexions 


\section{Github-Bookeditor}

\section{$\times \odot$ OERPUB GitHub book editor - Chromium}

몸 OERPUB GitHub book edil $\times$

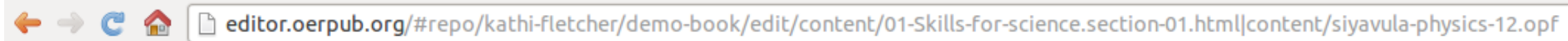

Table of Contents

E Editor: Scientific Theories

回 Save All

鼠 昆然

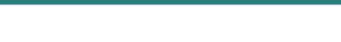

+ Add -

E Siyavula: Physics Gr 12

(a) Introduction to this editor an...

- Momentum and Impulse

- Introduction to Momentu.

D. Momentum

(1) Newton's Second Law Re..

- Science Basics

몬 Scientific Theories *

D. How Science Works

Tab Safety

$$
\theta=x^{2}+\sin y
$$

\section{General relativity}

Two-dimensional analogy of spacetime distortion generated by the mass of an object. Matter changes the geometry of spacetime, this (curved) geometry being interpreted as gravity. White lines do not represent the curvature of space but instead represent the coordinate system imposed on the curved spacetime, which would be rectilinear in a flat spacetime.

In general relativity, the effects of gravitation are ascribed to spacetime curvature instead of a force. The starting point for general relativity is the equivalence principle, which equates free fall with inertial motion, and describes free-falling inertial objects as being accelerated relative to noninertial observers on the ground.[8][9] In Newtonian physics, however, no such acceleration can occur unless at least one of the objects is being operated on by a force.

Einstein proposed that spacetime is curved by matter, and that free-falling objects are moving along locally straight paths in curved spacetime. These straight paths are called geodesics. Like Newton's first law of motion, Einstein's theory states that if a force is applied on an object, it would deviate from a geodesic. For instance, we are no longer following geodesics while standing because the mechanical resistance of the Earth exerts an upward force on us, and we are non-inertial on the ground as a result. This explains why moving along the geodesics in spacetime is considered inertial.

Einstein discovered the field equations of general relativity, which relate the presence of matter and the curvature of spacetime and are named
@oerpub
@kefletcher
http:/lbit.ly/fletcher-bib13
\#bib13 


\section{Principles: as much like word/gdocs as possible}

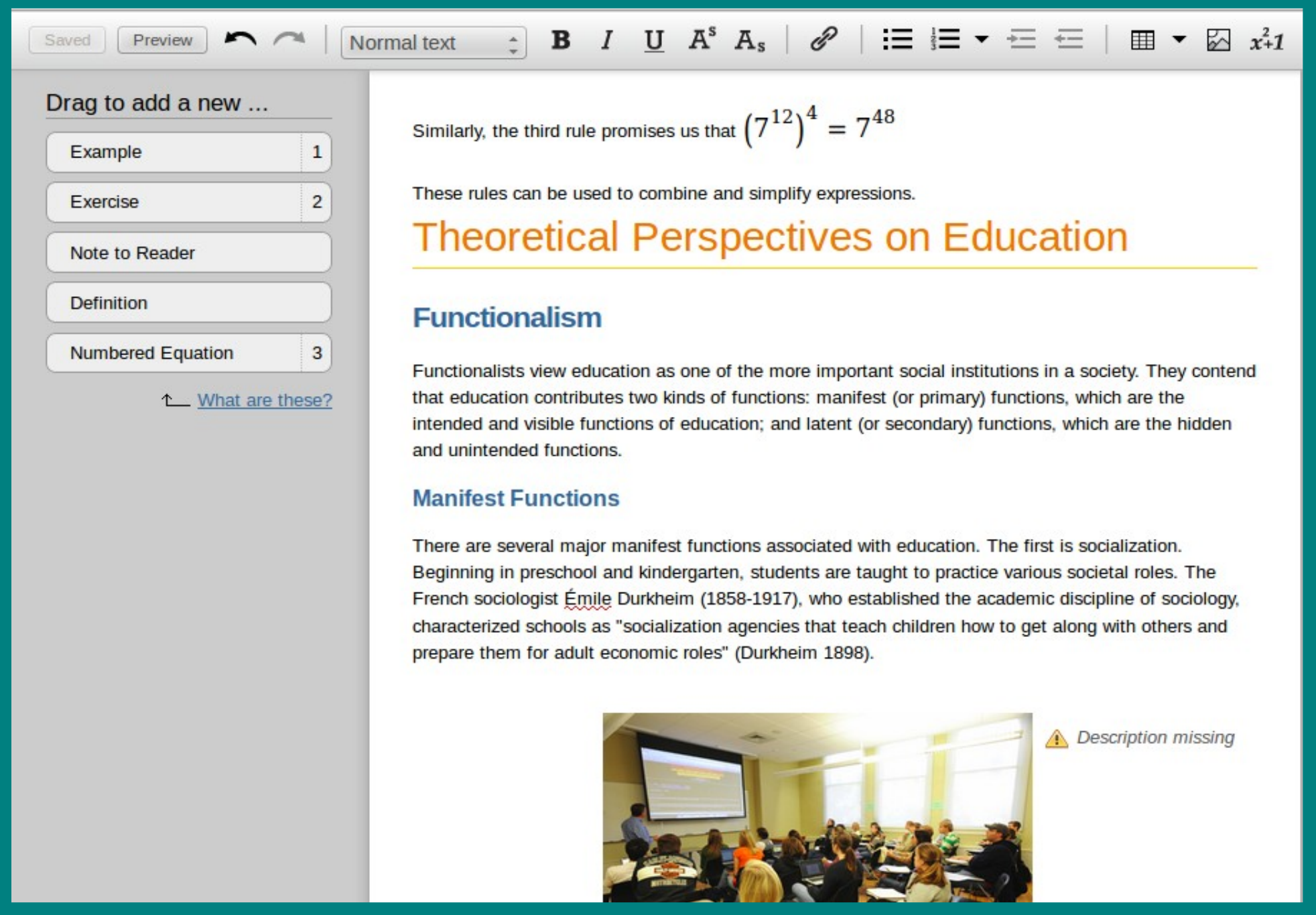




\section{Principle: support attribution}

\section{Insert image}
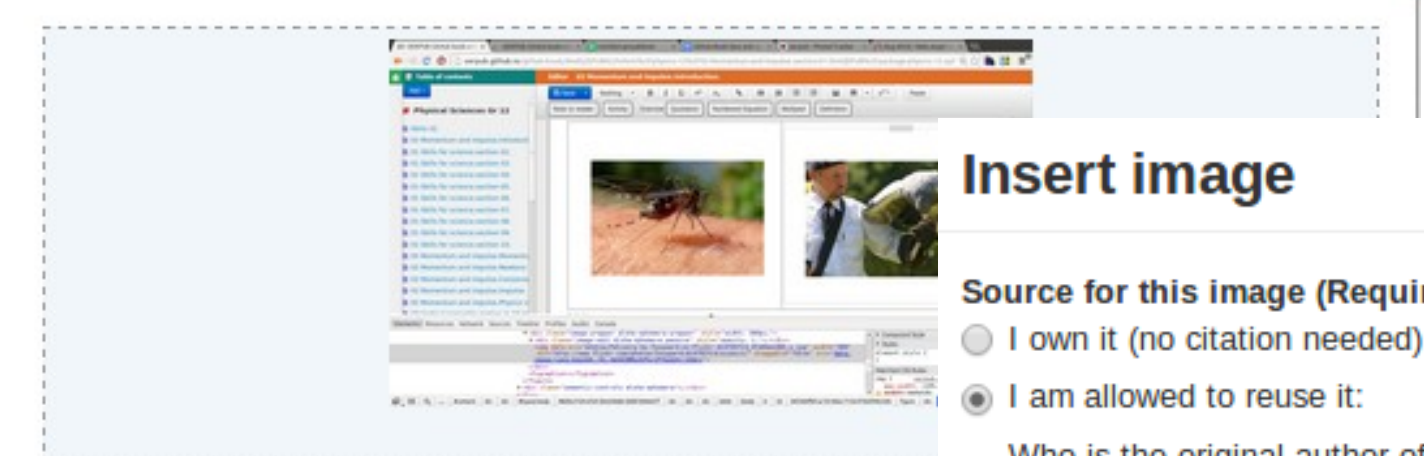

\section{Insert image}

Source for this image (Required)

I own it (no citation needed)

- I am allowed to reuse it:

Who is the original author of this image?

\section{Image title:}

Shows up above image

\section{Image caption:}

Shows up below image

4 Describe the image for someone who cannot see it. aloud, making it possible for visually impaired learners to und Enter description ..

\section{What organization owns this image?}

What is the original URL of this image?

\section{http://}

Permission to reuse

Choose a license

I don't know (skip citation for now)

\section{@oerpub




\section{Principle: born accessible Images, tables, mathematics}

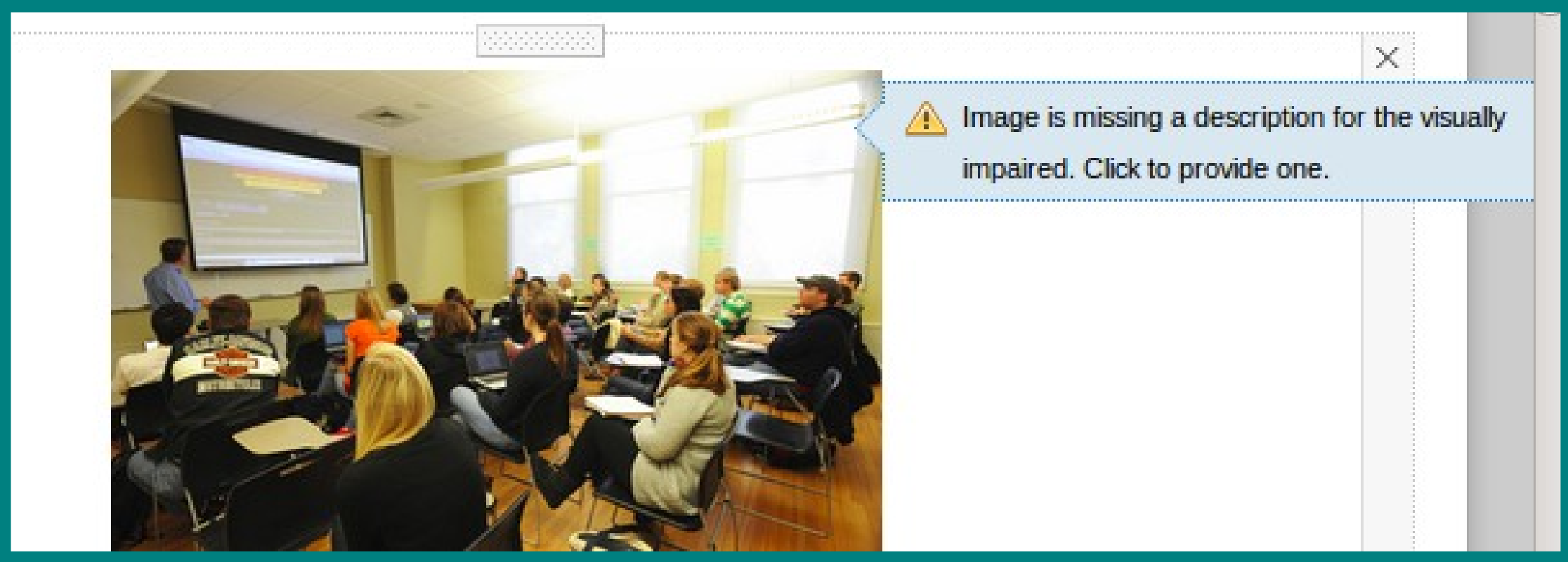

@oerpub @kefletcher http:Ilbit.lylfletcher-bib13 \#bib13 


\section{Principle: Mathematics editing support}

Editor: Scientific Theories

\begin{tabular}{|c|c|c|c|c|c|c|c|c|c|c|c|c|c|c|c|c|c|}
\hline Normal Text & - & B & $I$ & $\underline{\mathbf{U}}$ & $A^{S}$ & $\mathrm{~A}_{\mathrm{S}}$ & 8 & $\vdots \equiv$ & $\frac{1}{3} \equiv$ & 트 & EE & 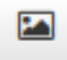 & 囲 & r & $x^{2+1}$ & 四 & \\
\hline \multicolumn{2}{|c|}{ Drag to add a new ... } & \multicolumn{3}{|c|}{ te to reader } & \multicolumn{2}{|c|}{ Activity } & \multicolumn{2}{|c|}{ Exercise } & \multicolumn{2}{|c|}{ Quotation } & \multicolumn{4}{|c|}{ Numbered Equation } & \multicolumn{2}{|c|}{ Multipart } & Definition \\
\hline
\end{tabular}

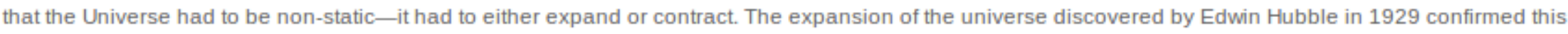
prediction.[15]

- The theory's prediction of frame dragging was consistent with the recent Gravity Probe B results.[16]

- General relativity predicts that lig University of Copenhagen collec $\quad \mid x^{\wedge} 2+y^{\wedge} 2=z^{\wedge} 2$ Iforall $x, y, z$ cluster edges, confirming the en

This is: $\bigcirc$ ASCIIMath LaTeX $\odot$ Plain text Copy Done
\[ x^{2}+y^{2}=z^{2} \forall x, y, z \]

Vojtak of the Niels Bohr Institute a ded to be red-shifted compared to

\section{Gravity and quantum mechanics}

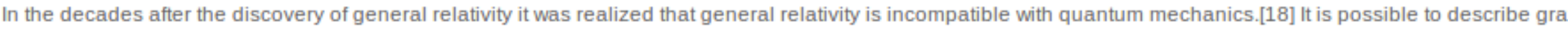

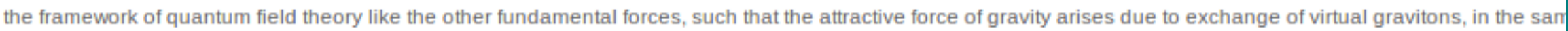

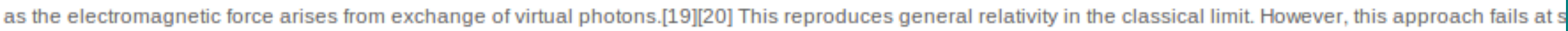

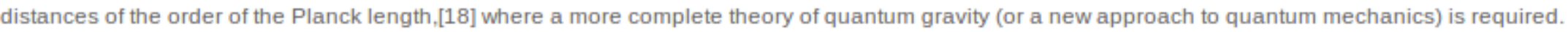

Atomic theory

Math Cheat Sheet: Copy the "code" that matches the display you want. Paste it into the math entry box above. Adjust as $\approx$ needed.

- Ascilmath

- LaTeX
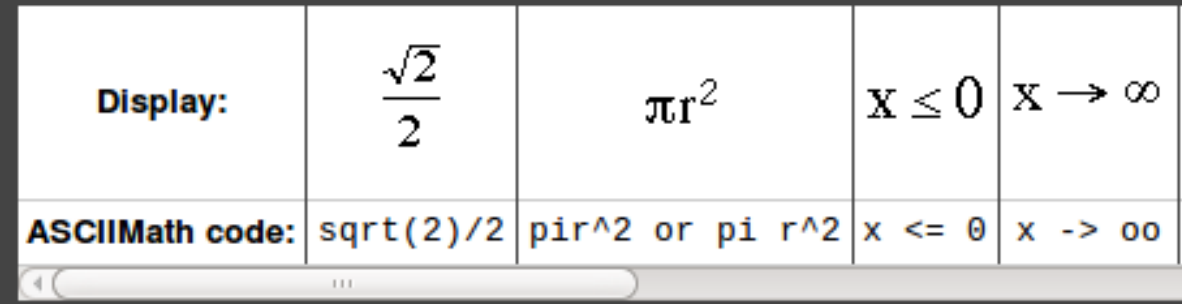

$$
\left(\frac{A+X}{2}, \frac{B+Y}{2}\right)
$$$$
\sum_{k=0}^{s-1}
$$

sum_ $\{k=0\}^{\wedge}\{s-1$

\section{@oerpub}

\section{@kefletcher}




\section{Principle: drag and drop examples, exercises, notes}

\begin{tabular}{l} 
器 OERPUB GitHub book edil $\times$ \\
\hline \\
\hline
\end{tabular}

\section{Momentum}

Momentum is a physical quantity which is closely related to forces. Momentum is a property which applies to moving objects, in fact it is mass in motion. If something has mass and it is moving then it has momentum.

- Definition Momentum

Drag this definition to another location

The linear momentum of a particle (object) is a vector quantity equal to the product of the mass of the particle (object) and its velocity.

The momentum (symbol $\vec{p}$ ) of an object of mass $m$ moving at velocity $v$ is:

$\vec{p}=m \vec{v}$

Momentum is directly proportional to both the mass and velocity of an object. A small car travelling at the same velocity as a big truck will have a smaller momentum than the truck. The smaller the mass, the smaller the momentum for a fixed velocity. If the mass is constant then the greater the velocity the greater the momentum. The momentum will always be in the same direction as the velocity because mass is a scalar not a vector.

\section{@oerpub}




\section{Principle: Support collaboration}

\section{(४) OERPUB GitHub book editor - Chromium}

吅 OERPUB GitHub book edi $x$

e $\rightarrow$ C editor.oerpub.org/\#repo/kathi-fletcher/demo-book/edit/content/01-Skills-for-science.section-01.html|content/siyavula-physics-12.opf

Table of Contents

Editor: Scientific Theories

Đ Save All

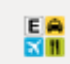
鼠

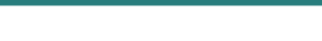

\section{+ Add -}

E Siyavula: Physics Gr 12

Q Introduction to this editor an...

- Momentum and Impulse

I Introduction to Momentu..

[) Momentum

을 Newton's Second Law Re..

- Science Basics

D Scientific Theories *

国 How Science Works

⿴囗十) Lab Safety

$$
\theta=x^{2}+\sin y
$$

\section{General relativity}

Two-dimensional analogy of spacetime distortion generated by the mass of an object. Matter changes the geometry of spacetime, this (curved) geometry being interpreted as gravity. White lines do not represent the curvature of space but instead represent the coordinate system imposed on the curved spacetime, which would be rectilinear in a flat spacetime.

In general relativity, the effects of gravitation are ascribed to spacetime curvature instead of a force. The starting point for general relativity is the equivalence principle, which equates free fall with inertial motion, and describes free-falling inertial objects as being accelerated relative to noninertial observers on the ground.[8][9] In Newtonian physics, however, no such acceleration can occur unless at least one of the objects is being operated on by a force.

Einstein proposed that spacetime is curved by matter, and that free-falling objects are moving along locally straight paths in curved spacetime. These straight paths are called geodesics. Like Newton's first law of motion, Einstein's theory states that if a force is applied on an object, it would deviate from a geodesic. For instance, we are no longer following geodesics while standing because the mechanical resistance of the Earth exerts an upward force on us, and we are non-inertial on the ground as a result. This explains why moving along the geodesics in spacetime is considered inertial.

\section{@oerpub @kefletcher http:/lbit.ly/fletcher-bib13 \#bib13}




\section{About github-bookeditor}

- Usability, accessibility

- Books stored on github

- Format EPUB3/HTML5 (TextbookHTML)

- Edit in the browser

- Open source (github.com/oerpub)

- Based on Aloha

- Embeddable, adaptable 


\section{View book on github}

\section{Contents of EPUB}

\section{IEB Physics Outline}

1. Introduction to this editor and project

2. 01 Vectors

3. 02 Kinematics

5. 04 MomentumAndImpuls

6. 05 WorkEnergyPower

7. 06 NewtonsLawUniversalGravitation

8. 07 Electrostatics

9. 08 ElectricCircuits

10. 09 Electrodynamics

11. 10 PhotoElectricEffectAndEmissionSpectra

12. template

\section{Siyavula: Physics Gr 12}

1. Introduction to this editor and project 2. Momentum and Impulse

1. Introduction to Momentum and Impulse

2. Momentum

3. Newton's Second Law Revisted 3. Science Basics

1. Scientific Theories

2. How Science Work

3. Lab Safety

\section{undefined}

\section{Introduction}

In Grade 10 we studied motion but not what caused the motion, in Grade 11 we learnt about forces and how they can alter the motion of an object. In this chapter we will focus on what happens when two bodies undergo a contact interaction and how their motion is affected. We learn more about how force and motion are related. We are introduced to two new concepts, momentum and impulse.

We can begin by considering some scenarios to set the context. Most people have some intuition for physics based on their everyday experiences but they haven't formalised it. We can use our intuitive answers to lead into more structured thinking about physical events.

Momentum transfer doesn't require a contact interaction but we won't consider any non-contact scenarios in this chapter.

Everyone has experienced a mosquito landing on their arm and it can happen quite unnoticed. Consider the case of a falcon landing on your arm (ignore the sharp claws for now). You would definitely notice, why? What makes a mosquito different to a falcon? Would you still notice if the mosquito flew the same way as a falcon, or if the falcon copied the flight of a mosquito before landing? You probably would still notice, but try to think about what makes them so different.

Table 1
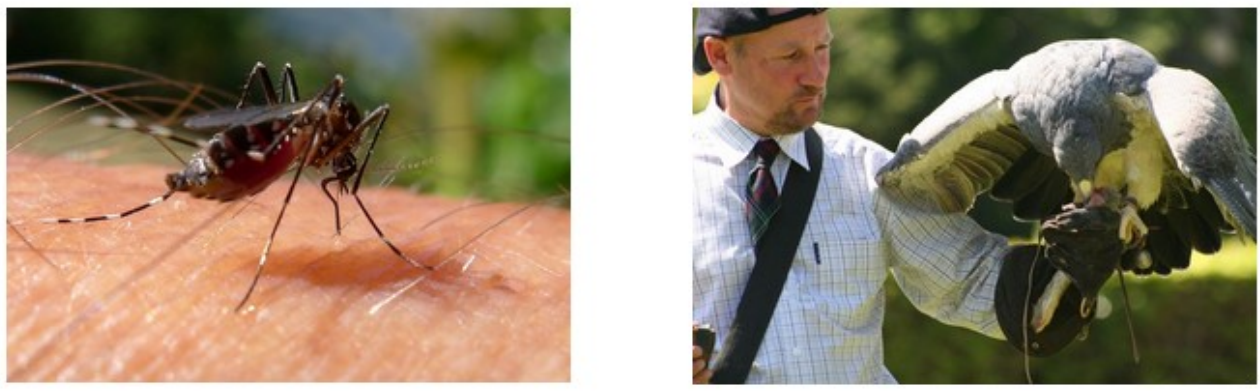

Look at a motorcycle, motorcar and truck. Which of them is more likely to result in less damage in a collision situation, why? What factors would you change to reduce potential damage. Table 2
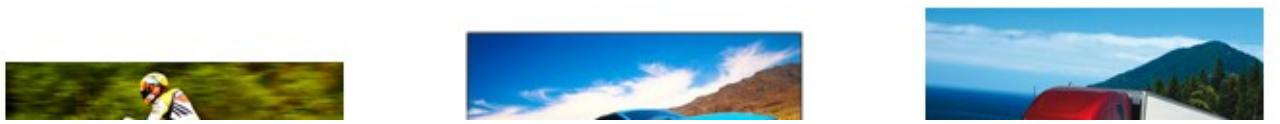

\section{@oerpub @kefletcher http:/lbit.lylfletcher-bib13 \#bib13}




\section{Services: PDF on demand}

\section{Recent Builds}

○ oerpub/demo-book

philschatz/books

philschatz/epub-anatomy

philschatz/test-book

oerpub/empty-book

philschatz/minimal-book

\section{oerpub/demo-book}

\section{↔ Download PDF}

\author{
Current Build History
}

Build Info

Rebuild PDF

\author{
Status: COMPLETED \\ Created: 5 hours ago \\ Duration: 8 seconds
}

Pulling remote updates

Already up-to-date.

Checking if mimetype file exists

\{ "msg" : "Reading file" , "path": "mimetype"\}

\{ "msg": : "Reading file", "path" : "META-INF/container.xml "\}

\{ "msg" : "jQuery built for file" , "path" : "META-INF/container. xml "\}

\{ "msg" : "Reading file", "path" : "content/siyavula-physics-12.opf"\}

\{"msg" : "jQuery built for file", "path" : "content/siyavula-physics-12.opf"\}

Reading Toc Navigation file

\{ "msg" : "Reading file", "path" : "content/siyavula-physics-12-nav.html "\}

\{"msg": "jQuery built for file", "path" : "content/siyavula-physics-12-nav. html "\}

\{ "msg" : "Reading file" , "path" : "content/Project-intro.html"\}

\{"msg" : "Reading file", "path" : "content/02-Momentum-and-impulse. section-01.html "\}

\{ "msg" : "Reading file", "path" : "content/02-Momentum-and-impulse. section-02.html "\}

\{"msg" : "Reading file", "path" : "content/02-Momentum-and-impulse. section-03.html" \}

\{"msg" : "Reading file", "path" : "content/01-Skills-for-science. section-01.html"\} 


\section{Services: PDF on demand}

has a circular orbit. Using the equation for the circumference, $\mathrm{C}$, of a circle in terms of its radius, we can determine the distance travelled by the Moon in one orbit:

$$
\begin{aligned}
C & =2 \pi r \\
& =2 \pi\left(3,844 \times 10^{8}\right) \\
& =2,42 \times 10^{9} \mathrm{~m}
\end{aligned}
$$

Combining the distance travelled by the Moon in an orbit and the time taken by the Moon to complete one orbit, we can determine the magnitude of the Moon's velocity or speed,

$$
\begin{aligned}
v & =\frac{\Delta x}{\Delta t} \\
& =\frac{C}{T} \\
& =\frac{2,42 \times 10^{9} \mathrm{~m}}{2,36 \times 10^{6} \mathrm{~s}} \\
& =1,02 \times 10^{3} \mathrm{~m} \cdot \mathrm{s}^{\wedge}\{-1\} .
\end{aligned}
$$

Finally calculate the momentum and quote the answer

The maonitude of the Monn's momentum is.

\section{@oerpub \\ @kefletcher




\section{Other versions of the editor: remix.oerpub.org}

\section{OERPUB Connexions Importer}

\section{Create, edit, and adapt content in Connexions}

Choose one of the following to create, import, or edit the contents of a new or existing module.

Module editor

\begin{tabular}{l|l} 
Create a new module Edit an existing module $~$
\end{tabular}

Or use one of our importer tools

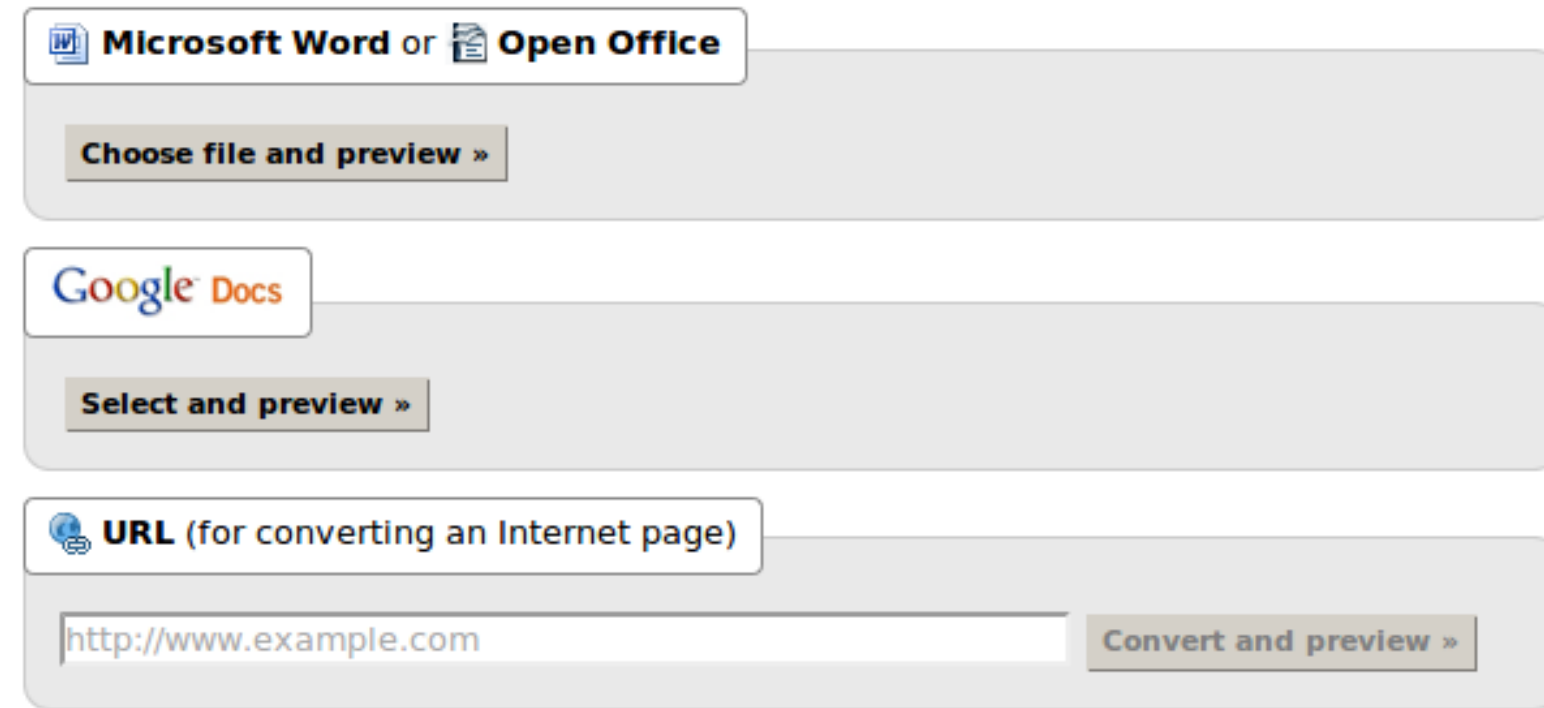

Or try our alpha importer tools

Microsoft Presentation - Open/Libre Office Impress 


\section{Other versions of the editor: remix.oerpub.org}

X OeRPuB Connexions Importer

« Back: Return to start page
You are logged in as: anonymous. Sign ou

Next: Describe module »
Saved
$\sim \sim$
Normal Text $\quad$ |
B $I \quad \underline{\mathrm{U}} \quad \mathrm{A}^{\mathrm{s}} \quad \mathrm{A}_{\mathrm{s}}$

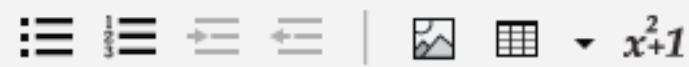
Add a new... .

\section{Drag to add a new ...}

Note to reader

Activity

Exercise

Quotation

Numbered Equation

\section{Theoretical Perspectives on Education}

\section{Functionalism Demo}

Functionalists view education as one of the more important social institutions in a society. They contend that education contributes two kinds of functions: manifest (or primary) functions, which are the intended and visible functions of education; and latent (or secondary) functions, which are the hidden and unintended functions.

\section{Manifest Functions}

There are several major manifest functions associated with education. The first is socialization. Beginning in preschool and kindergarten, students are taught to practice various societal roles. The French sociologist Émile Durkheim (1858-1917), who established the academic discipline of sociology, characterized schools as "socialization agencies that teach children how to get along with others and prepare them for adult economic roles" (Durkheim 1898).

testing.oerpub.org/transforms/anonymous-20131025-060027/\# 


\section{Connexions' version of the editor}

\begin{tabular}{|c|c|c|c|c|c|c|c|c|c|c|c|c|}
\hline Add - & Nothing & 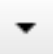 & B & $I$ & $\underline{\mathbf{U}}$ & 0 & $x^{2}$ & Note & $\geqq$ & $\frac{1}{\frac{1}{3}} \equiv$ & 厈 & 트 \\
\hline
\end{tabular}

\section{E Moby Dick}

(Back to Workspace)

- 国 The Carpet-Bag

国 Loomings

$\square$ The Spouter-Inn.

- $\square$ Breakfast.

$\square$ The Street.

$\square$ The Pulpit.

$\square$ The Sermon.

$\square$ A Bosom Friend.

$\square$ Epilogue

$\square$ Copyright Page

\section{Title: Chapter 1. Loomings}

Metadata

Roles

Call me Ishmael.

Some years ago—never mind how long precisely—having little or no money in my purse, and nothing particular to in There now is your insular city of the Manhattoes, belted round by wharves as Indian isles by coral reefs-commerce Circumambulate the city of a dreamy Sabbath afternoon. Go from Corlears Hook to Coenties Slip, and from thence, But look! here come more crowds, pacing straight for the water, and seemingly bound for a dive. Strange! Nothing w Once more. Say you are in the country; in some high land of lakes. Take almost any path you please, and ten to one But here is an artist. He desires to paint you the dreamiest, shadiest, quietest, most enchanting bit of romantic lands Now, when I say that I am in the habit of going to sea whenever I begin to grow hazy about the eyes, and begin to b

\section{@oerpub




\section{Another adaptation: DHWriter}

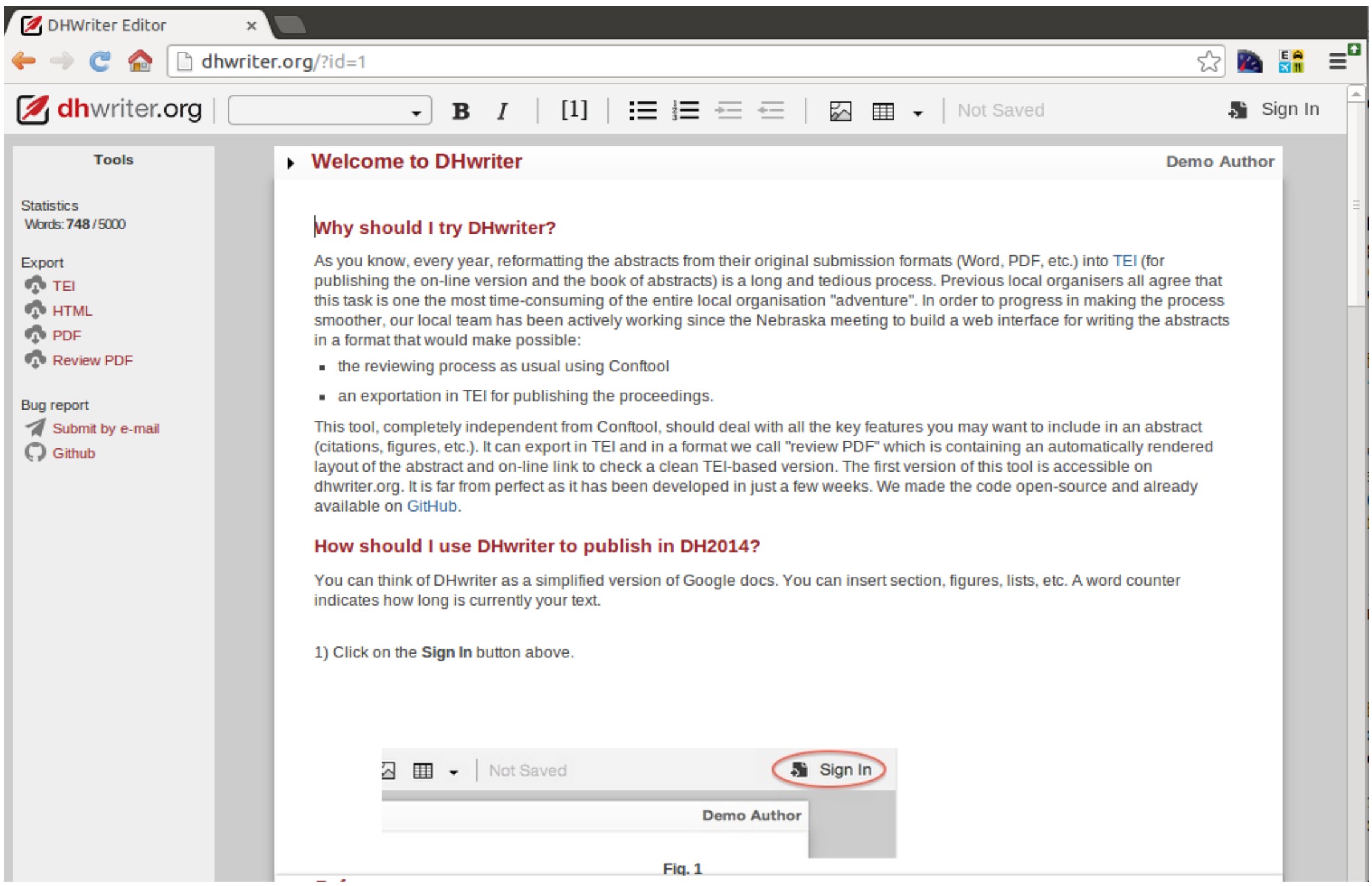

\section{@oerpub




\section{Try it out}

\section{Bookeditor (alpha): (Chrome only right now)}

If you don't have a github account, $x$ out of the login and you can be anonymous

Demo book opens and has some more information and links editor.oerpub.org

or

oerpub.github.io/github-bookeditor

Developers:

github.com/oerpub/github-bookeditor github.com/oerpub/Aloha-Editor github.com/philschat>Indf_ri

SHUTTLEWORTH FELLOW

C O N N E I T O ONX.ORG

\begin{tabular}{|l|l|l|}
\hline $\mathbf{O}$ & $\mathbf{E}$ & $\mathbf{R}$ \\
\hline $\mathbf{P}$ & $\mathbf{U}$ & $\mathbf{B}$ \\
\hline
\end{tabular}




\section{Hackday Tomorrow!}

October 26, San Francisco

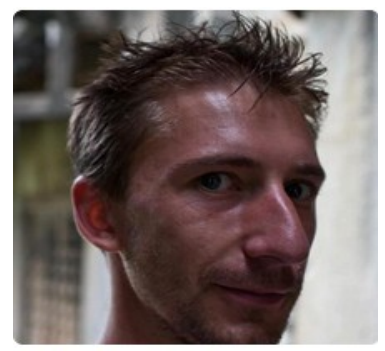

Philip Schatz philschatz
Find Phil - key designer, developer cnx.org

Help us integrate EPUBjs and Hypothes.is

Use vagrant to get a developer instance up and explore your own ideas to extend the editor.

SHUTTLEWORTH FELLOW

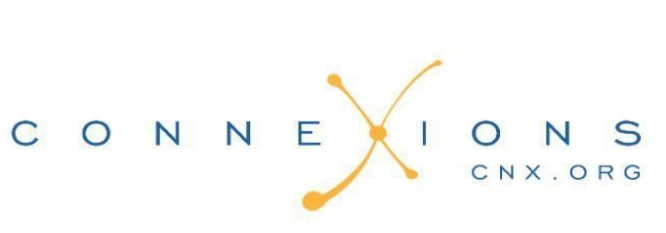

\begin{tabular}{|l|l|l|}
\hline O & E & R \\
\hline $\mathbf{P}$ & $\mathbf{U}$ & $\mathbf{B}$ \\
\hline
\end{tabular}

